\title{
Geometric Calibration of Low-Cost Flatbed Scanners for Large Scale Mapping Applications
}

\author{
Ahmed F. Elaksher ${ }^{1}$, Tarig Ali ${ }^{2}$ \\ ${ }^{1}$ Civil Engineering Department, College of Engineering, Cal Poly Pomona University, Pomona, USA \\ ${ }^{2}$ Department of Civil Engineering, College of Engineering, American University of Sharjah, Sharjah, UAE \\ Email: atarig@aus.edu
}

How to cite this paper: Elaksher, A.F. and Ali, T. (2018) Geometric Calibration of Low-Cost Flatbed Scanners for Large Scale Mapping Applications. Modern Instrumentation, 7, 11-23.

https://doi.org/10.4236/mi.2018.72002

Received: December 20, 2017

Accepted: February 5, 2018

Published: February 8, 2018

Copyright () 2018 by authors and Scientific Research Publishing Inc. This work is licensed under the Creative Commons Attribution International License (CC BY 4.0).

http://creativecommons.org/licenses/by/4.0/

(c) (i) Open Access

\begin{abstract}
Scanning hardcopy non-metric images is one of the most important sources in digital mapping. Low-cost scanners are still widely used in many applications as they can produce digital images of comparable precisions to those produced by expensive professional scanners. Yet, inexpensive scanners introduce geometrical distortions in the measured image coordinates that must be assessed and compensated before using their products for further analysis. In this article, several 2D-to-2D transformation models were investigated to calibrate flatbed scanners with different resolutions and sizes. We evaluated the potential of each model using two gridded-crosses plotted on high-quality transparent sheets. Control coordinates were provided through a photogrammetric analytical plotter. After scanning the sheets, least squares matching was applied to determine the precise locations of the crosses. By comparing the control coordinates and those estimated from digitized images, it was found that the mathematical model based on the projective transformation gives the best results for standardizing the geometric properties of flatbed scanners. The results show that scanning resolution of $2400 \mathrm{dpi}$ achieves the requirements for large-scale mapping applications.
\end{abstract}

\section{Keywords}

Flatbed Scanners, Projective Transformation, Affine Transformation, Photogrammetry, GIS

\section{Introduction}

Scanners are an essential part of softcopy mapping systems. Despite the rapid dependence on digital cameras, scanners have been used for several years as a source of digital images. Several developing countries are still using analog cam- 
eras for their mapping activities, [1] [2] [3] [4]. Additionally, converting historical films to digital format is still needed to incorporate them into (Geographic Information Systems) GIS databases for change detection, erosion, sediment transportation, and other tasks [5]-[11].

There are several aspects and requirements for scanners to be employed in digital mapping. Their illumination must be adequate to reach the best radiometric quality and Signal-to-Noise-Ratio (SNR). They should have an extended dynamic range to discriminate between neighboring pixels. Their pixel size, geometric resolution, should be fine enough for interpretation of fine details and measurement of small signalized objects. Since the late 1980s, high-end photogrammetric scanners have been employed in scanning analog photos and hardcopy maps, [12]. In recent years, low-cost digital mapping systems have been heavily employed in surveying and mapping applications, [13]. These systems rely mainly on desktop scanners, particularly flatbed scanners that are of less quality than photogrammetric scanners. Flatbed scanners typically employ one or more linear CCDs, and move in a direction perpendicular to the CCD. The imaging architectures and properties of flatbed scanners are outlined in [14].

The possibility of losing the full radiometric spectrum or the fine spatial details during scanning process is an inherent drawback of converting photographs into digital formats, [15]. Hence, it is vital to study the effects of this process on the geometric accuracy and visual quality of the original image. Several studies have looked into the radiometric characteristics of flatbed scanners, ([16] [17] [18]). The radiometric performance of scanners could be affected by noise level, linearity, dynamic range, effect of illumination variation, and presence of artifacts or blemished pixels [19]. The geometric characteristics of the photogrammetric scanners were studied in [20]. He concluded that there is critical need to find the best approach for the geometric calibration of the flatbed scanners.

The digitizing analog aerial images with low-cost scanners was studied in [21]. Results were compared with those captured by high-end photogrammetric scanners. It was found that small-format scanners could achieve the accuracy requirements for topographic mapping at a scale of 1:5000. The suitability of nonprofessional scanners for aerial photogrammetric mapping was addressed in [22]. He revealed that these scanners could worsen the horizontal and vertical accuracies by about $40 \%$ from the Leica BC2 analytical plotter. Yet, he concluded that the achieved precision is suitable for planimetric maps of 1:5000 scale and to produce contours of one-meter interval. A procedure was outlined in [23] to convert flatbed scanners to traceable table to measure 2D coordinates. For calibrating the scanner, a calibration parameter was added to the transformation model and estimated through the measure of a line scale. After calibration, it was shown that measured elements expanded at the order of the resolution of the flatbed scanner.

In [24], it was shown that the position of the scanned object relative to the scanner and the scanning direction cause major variations in the dimensions of 
the images of scanned objects. Insignificant periodic errors were found in [25] parallel to the linear detector in the scanner. However, they revealed errors in the range of $0.03 \mathrm{~mm}$ to $0.05 \mathrm{~mm}$ in the direction perpendicular to the detector for a $0.01 \mathrm{~mm}$ resolution scanner. A procedure to estimate uncertainties in scanned elliptical shapes was unveiled in [26]. The relative uncertainty in dimensional measurements with a flatbed scanner at the $95 \%$ confidence level is under $0.1 \%$ was presented in [27]. An algorithm to correct for the parabola effect in flatbed scanners for dosimetry applications was outlined in [28].

Besides these studies, other researchers addressed the geometric properties of digitized documents through different approaches. A content independent approach to compensate for the geometric distortions in document-images captured by digital cameras, which is very useful for scanned documents was proposed in [29]. Firstly, they recover two spatial curves representing the 3D page surface. A 3D developable surface is then used to fit the curves. Finally, through a system of ordinary differential equations, the developable surface is flattened onto a plane. Stereo measurement procedures were applied in [30] to correct for geometric distortion in document images. The geometric and photometric distortions in 3D shapes of book surfaces from the shading information in their scanned image were compensated in [31]. A book de-warping system to remove the perspective and geometric distortions automatically from single images by boundary-based 3D surface reconstruction was presented in [32].

\section{Coordinate Transformation}

The scanner is a device that converts hardcopy images to digital form stored by the computer. This is done by dividing the image into pixels. The sensor of the scanner scans the image from one side to the other, converting the gray levels of pixels to numerical values. The positions of the pixels are referenced to a coordinate system that has its origin at the upper left corner of the image; the (R) axis aims downward, while the $(\mathrm{C})$ axis aims towards the right direction Figure 1. The resolution of the scanner is the ratio between the numbers of pixels to the corresponding area of the hardcopy image. A typical scanner has a resolution that ranges from $300 \mathrm{dpi}$ up to $4800 \mathrm{dpi}$. This indicates that each pixel has an approximate dimension of $84 \mu \mathrm{m}$ to $5 \mu \mathrm{m}$. This is very comparable with most photogrammetric scanners, [33]. Yet low-cost scanners are subject to additional geometric errors. Different sources for these errors such as radial and tangential lens distortions, CCD misalignment, and uniformity of geometric resolution were highlighted in [34]. Therefore, the positions of the points could be corrupted causing the distances between these points to change.

During the scanning process, photo coordinates, in the hardcopy photos, are transferred into the scanner coordinates, in the digital images. Several mathematical models have been utilized for such coordinate transformation. They vary from simple changes of position (without changing in shape or size), to a uniform change in scale (without changing in shape), to a change in the shape and 


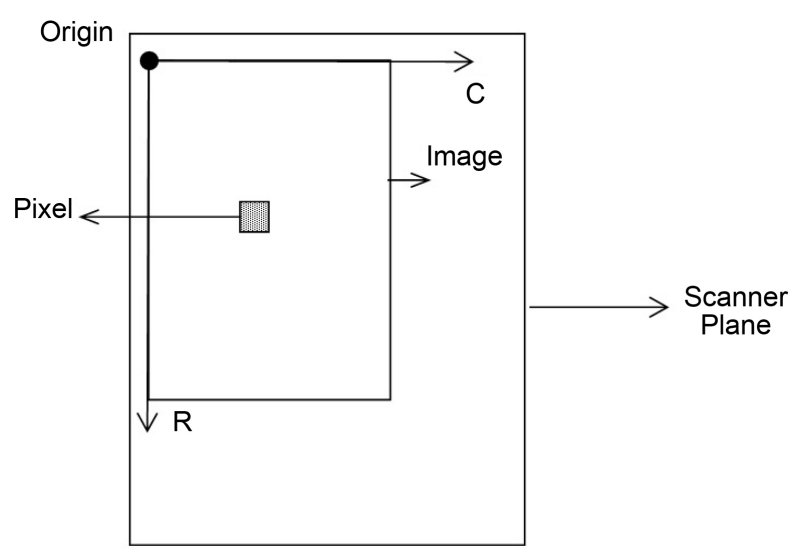

Figure 1. Scanner coordinate system, the scanner sweeps from top to bottom, C: Column coordinate, R: Raw coordinate, Origin $(1,1)$.

the size. In the following sections, a brief description for those applied in this research is presented.

\subsection{Conformal Transformation (Model 1)}

The conformal transformation is a linear transformation that relates the photo and scanner systems through a rotation $(\theta)$, a uniform scale change (S), and a translation $\left(X_{o}\right.$, and $\left.Y_{o}\right)$. The mathematical form of the model from system (1) to system (2) is:

$$
\begin{gathered}
\text { Scale } \\
X_{2}^{\prime}=S * X_{1} \\
Y_{2}^{\prime}=S * Y_{1} \\
\text { Rotation } \\
X_{2}^{\prime \prime}=X_{2}^{\prime} \cos \theta-Y_{2}^{\prime} \sin \theta \\
Y_{2}^{\prime \prime}=X_{2}^{\prime} \sin \theta+Y_{2}^{\prime} \cos \theta \\
\text { Shift } \\
X_{2}=X_{2}^{\prime \prime}+X_{o} \\
Y_{2}=Y_{2}^{\prime \prime}+Y_{o} \\
X_{2}=S * X_{1} \cos \theta-S * Y_{1} \sin \theta+X_{o} \\
Y_{2}=S * X_{1} \sin \theta+S * Y_{1} \cos \theta+Y_{o} \\
{\left[\begin{array}{ll}
X_{2} \\
Y_{2}
\end{array}\right]=S *\left[\begin{array}{ll}
\cos \theta & -\sin \theta \\
\sin \theta & \cos \theta
\end{array}\right]\left[\begin{array}{l}
X_{1} \\
Y_{1}
\end{array}\right]+\left[\begin{array}{l}
X_{o} \\
Y_{o}
\end{array}\right]}
\end{gathered}
$$

\subsection{Affine Transformation (Model 2)}

The affine transformation is a linear transformation similar to the conformal transformation but with two scale factors, a scale factor in the x-direction $\left(S_{x}\right)$ and a scale factor in the $y$-direction $\left(S_{y}\right)$. This represents non-constant shrinkage or elongation in the two directions. The mathematical form of the model from system (1) to system (2) is: 


\subsection{Projective Transformation (Model 3)}

The projective model represents a projection between two planes. The transformation between these two planes is expressed by two fractional linear equations that contain eight parameters. A relationship is established by which coordinates of points in plane (1) will be transformed to corresponding points in plane (2), Figure 2. The projective transformation is:

\subsection{Second Order Transformation (Model 4)}

This model is a polynomial of second order of the $X$ and $Y$ coordinates. The mathematical model is:

$$
\begin{aligned}
X_{2}= & a_{1}+a_{2} * X_{1}+a_{3} * X_{1}^{2}+a_{4} * X_{1} * Y_{1}+a_{5} * Y_{1}+a_{6} * Y_{1}^{2} \\
& +a_{7} * X_{1}^{2} * Y_{1}^{2}+a_{8} * X_{1}^{2} * Y_{1}+a_{9} * X_{1} * Y_{1}^{2} \\
Y_{2}= & b_{1}+b_{2} * X_{1}+b_{3} * X_{1}^{2}+b_{4} * X_{1} * Y_{1}+b_{5} * Y_{1}+b_{6} * Y_{1}^{2} \\
& +b_{7} * X_{1}^{2} * Y_{1}^{2}+b_{8} * X_{1}^{2} * Y_{1}+b_{9} * X_{1} * Y_{1}^{2}
\end{aligned}
$$

A least squares adjustment model, Equation (1), is then built to relate the coordinates in both systems. The transformation parameters are then found through Equation (2). Least squares optimization is guaranteed to converge after few iterations.

$$
\begin{gathered}
\boldsymbol{v}+\boldsymbol{B} \boldsymbol{\Delta}=\boldsymbol{f} \\
\boldsymbol{\Delta}=\left(\boldsymbol{B}^{\mathrm{T}} \boldsymbol{B}\right)^{-1}\left(\boldsymbol{B}^{\mathrm{T}} \boldsymbol{f}\right)
\end{gathered}
$$

where $v$ is the residual vector, $B$ is the coefficient matrix of the unknowns in the observation equations, $\Delta$ is the correction vector for the unknown parameters, i.e. the transformation parameters, $f$ is the coefficient vector of the observation equations.

\section{Experiments and Results}

\subsection{Reference Datasets}

We tested two precise hardcopy grids preparing by AutoCAD, Figure 3. The two

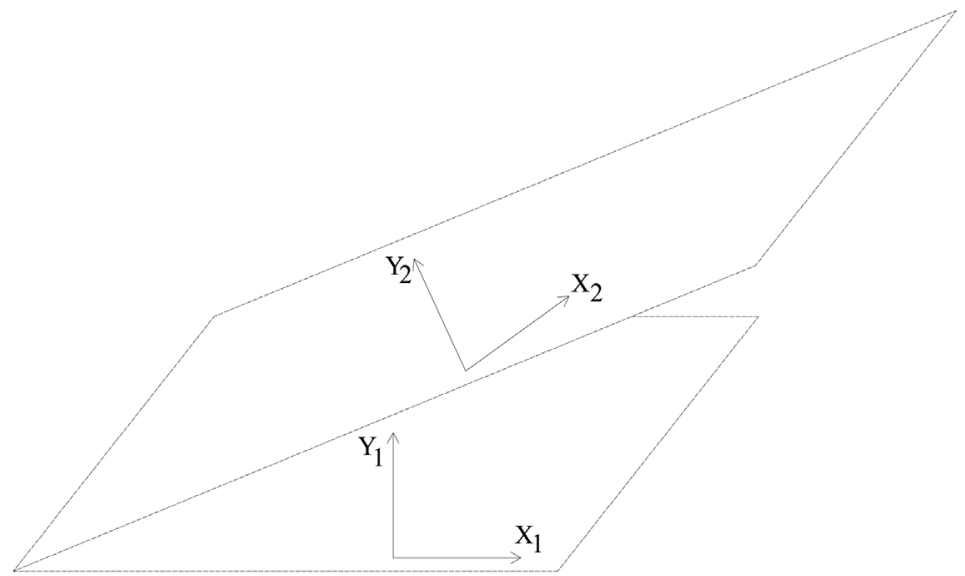

Figure 2. Projective transformation model. 


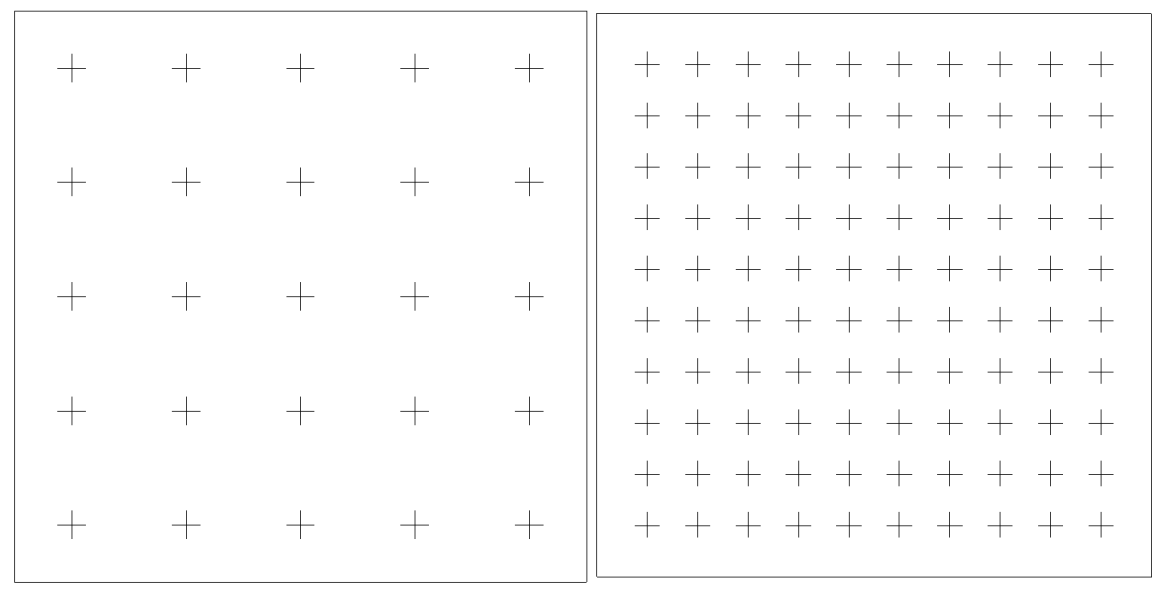

Figure 3. Grid plates for geometric tests and calibration.

grids were plotted on a high-quality plastic transparent sheets by a high-quality printer. The positions of the points on the hardcopy sheets were measured using a BC3 analytical plotter. The analytical plotter has a module that deals with only one image to be used as a comparator to give the image coordinates of targets in one image. Coordinates were measured three times and the averages of the coordinates were utilized for this research. The image coordinates of nine control points in each case are used with their AUTOCAD coordinates to find the transformation parameters of a projective transformation between the hardcopy coordinate system and the AUTOCAD coordinate system. The resulting parameters are then used to transfer the coordinates of other points to the AUTOCAD coordinate system and their Root Mean Square Errors (RMSEs) were estimated. The RMSEs in the $X$ and $Y$ directions were about $3 \mu \mathrm{m}$. Therefore, the coordinates measured by the analytical plotter serve as the reference corrected coordinates for the hardcopy image.

\subsection{Scanning Experiments}

To evaluate the scanning accuracy as well as to assess the most appropriate model that minimizes geometric distortions, several experiments were conducted with two scanners at different resolutions. The first scanner was a HP Scanjet G3110 scanner with a maximum scanning resolution of $4800 \times 9600 \mathrm{dpi}$. The second was a Mustek 2400S A3 large format $(299 \mathrm{~mm} \times 427 \mathrm{~mm})$ scanner with a maximum scanning resolution of $2400 \times 2400 \mathrm{dpi}$ without software interpolation. Different scanning resolutions, numbers of control points, and positions were tested.

A Least Square Matching (LSM) algorithm swept the image to estimate the image coordinates of the grid points. The template was selected to be a cross of $51 \times 51$ pixels. For each pixel, the template was centered on the pixel and then convolved with the neighboring pixels and the correlation between the template and the image was computed. To achieve sub-pixel accuracy, the template was displaced with increments of 0.25 pixel. The template was shrinkage and en- 
larged by ratios of $25 \%, 50 \%, 150 \%$, and $200 \%$. Additionally, the template was rotated every 150 to account for changes in the orientations of the grid. The outputs after applying the LSM algorithm are score images, i.e. for each pixel a score is assigned based on the correlation between the template and the neighboring pixels. We then searched for peaks in the score images and detected the locations of the intersections of the grid points in the scanned images. These locations and their correspondences measured by the analytical plotter were employed to calculate the transformation parameters for the four mathematical models. Next, the transformation parameters were applied to convert the coordinates of the check points in the scanned images to the analytical plotter coordinate system.

\subsection{Results and Analysis}

The RMSEs for the discrepancies between the transferred coordinates and those measured by the analytical plotter were then computed. The RMSEs are analyzed to find the optimal mathematical model to be adopted to compensate for the geometric distortion of the scanners. Figure 4 summarizes the results for the HP scanner while those for the Mustek scanner are presented in Figure 5. Results in both figures are reported with 10 control points and 15 check points while the grid plate is placed at the upper left corner of the scanners. For both scanners, it is apparent that the precisions in the $X$ direction, parallel to the linear CCD, is better than those in the $Y$ direction, scanning direction. In addition, as the resolution increases, the RMSEs goes down. The projective transformation model for both scanners achieved the least RMSEs in both $X$ and $Y$ directions. Insignificant

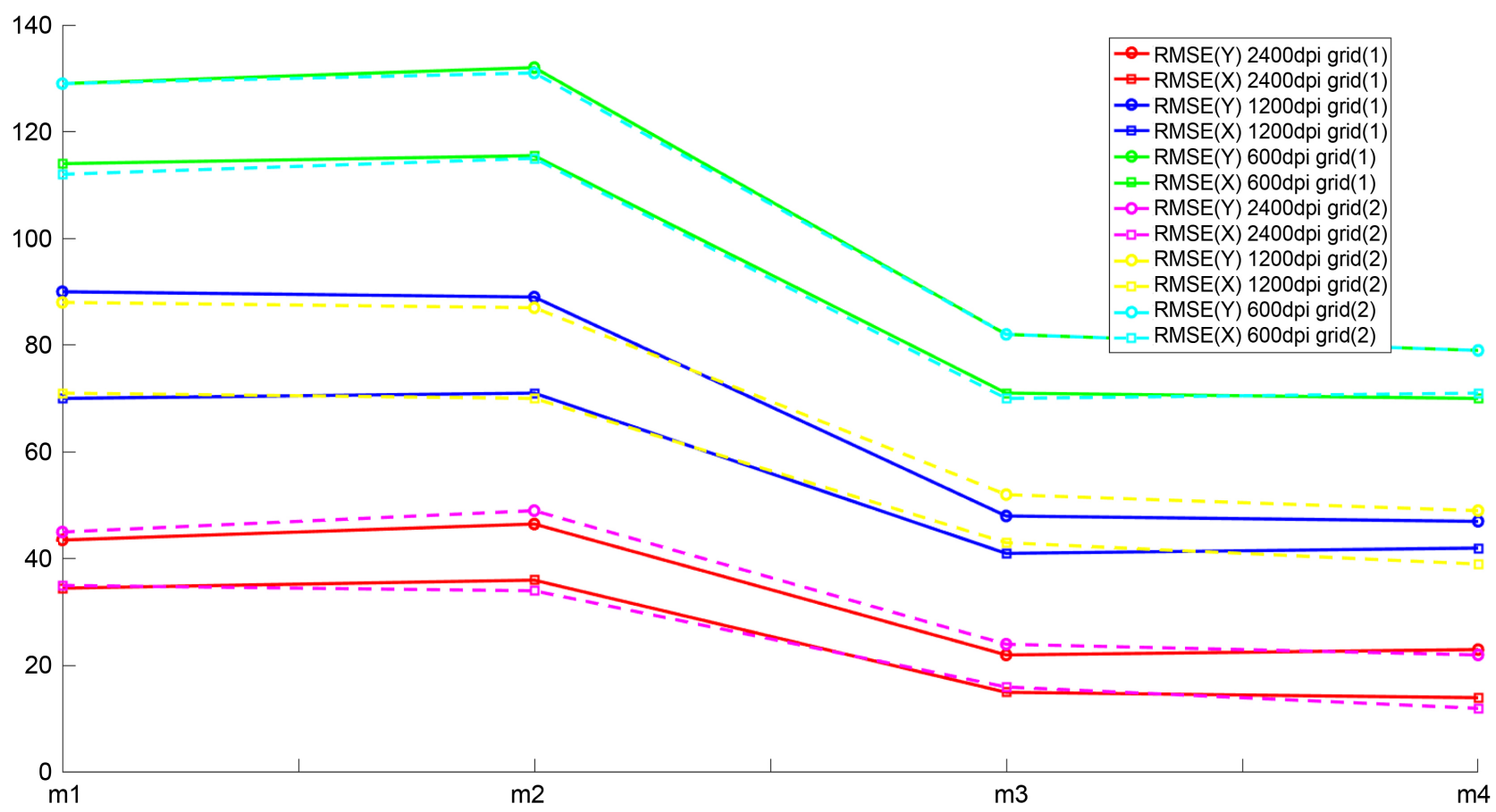

Figure 4. RMSEs for the HP scanner, mi: transformation model (i). 


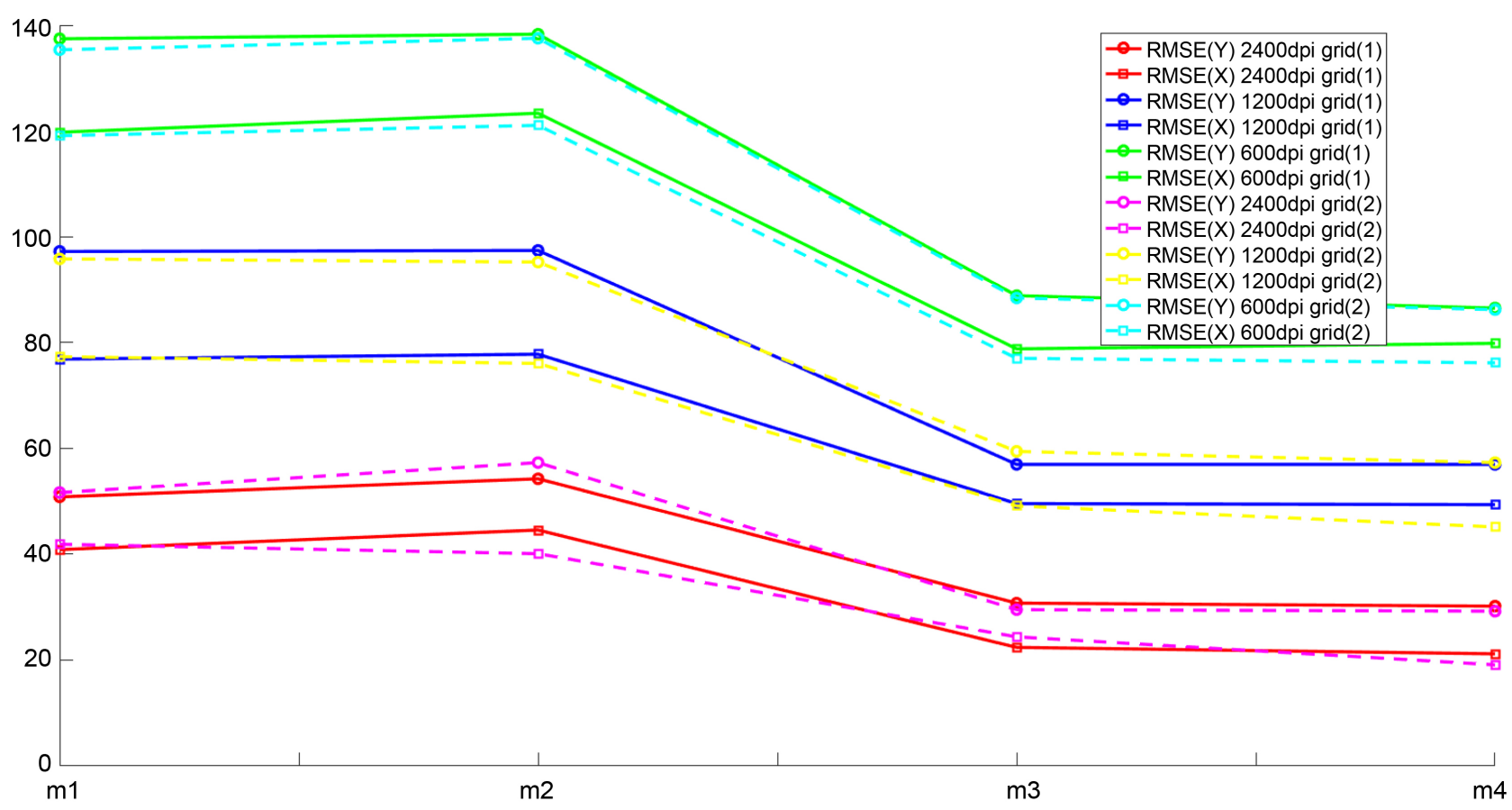

Figure 5. RMSEs for the Mustek scanner, mi: transformation model (i).

variations were observed when the second order transformation model was used, despite the increase in the number of parameters (ten).

The RMSEs didn't change for the same resolution, direction, and transformation model between both grid plates. With the projective transformation model, the RMSEs are within 1.5 to two times the resolution for the HP scanner and two to three times the resolution for the Mustek scanner. Figure 6 shows the effect of changing the number of control points on the RMSEs of the projective transformation model for both scanners with the 100-point grid plate scanned at 2400 dpi. With 10 or more control points, results for both scanners were stable and no improvements were seen beyond those for the 10 control points. This was noticed in both the $X$ and $Y$ directions. To test the uncertainties over the entire scanning field, we placed the 100-point grid plate at over different locations over the scanning glass. For each grid point, the RMSEs in the $X$ and $Y$ directions were estimated. The averages of the RMSEs in both $X$ and $Y$ directions are reported in Table 1 with $2400 \mathrm{dpi}$. The table shows that the RMSEs are similar to those estimated for the confined areas.

To evaluate the reliability of the scanning process for GIS applications, a set of aerial photos were scanned with different resolutions. Distances between twenty well-defined points were measured on one scanned photo and compared with those measured on the ground, Figure 7. The discrepancies between these distances are summarized in Table 2. The table also shows the Ground Sample Distance (GSD), i.e. the size of the pixel on the ground. For the 600 dpi resolution, discrepancies for both scanners fulfill the American Society for Photogrammetry and Remote Sensing, [35], 1:2000 map scale requirement. For the 


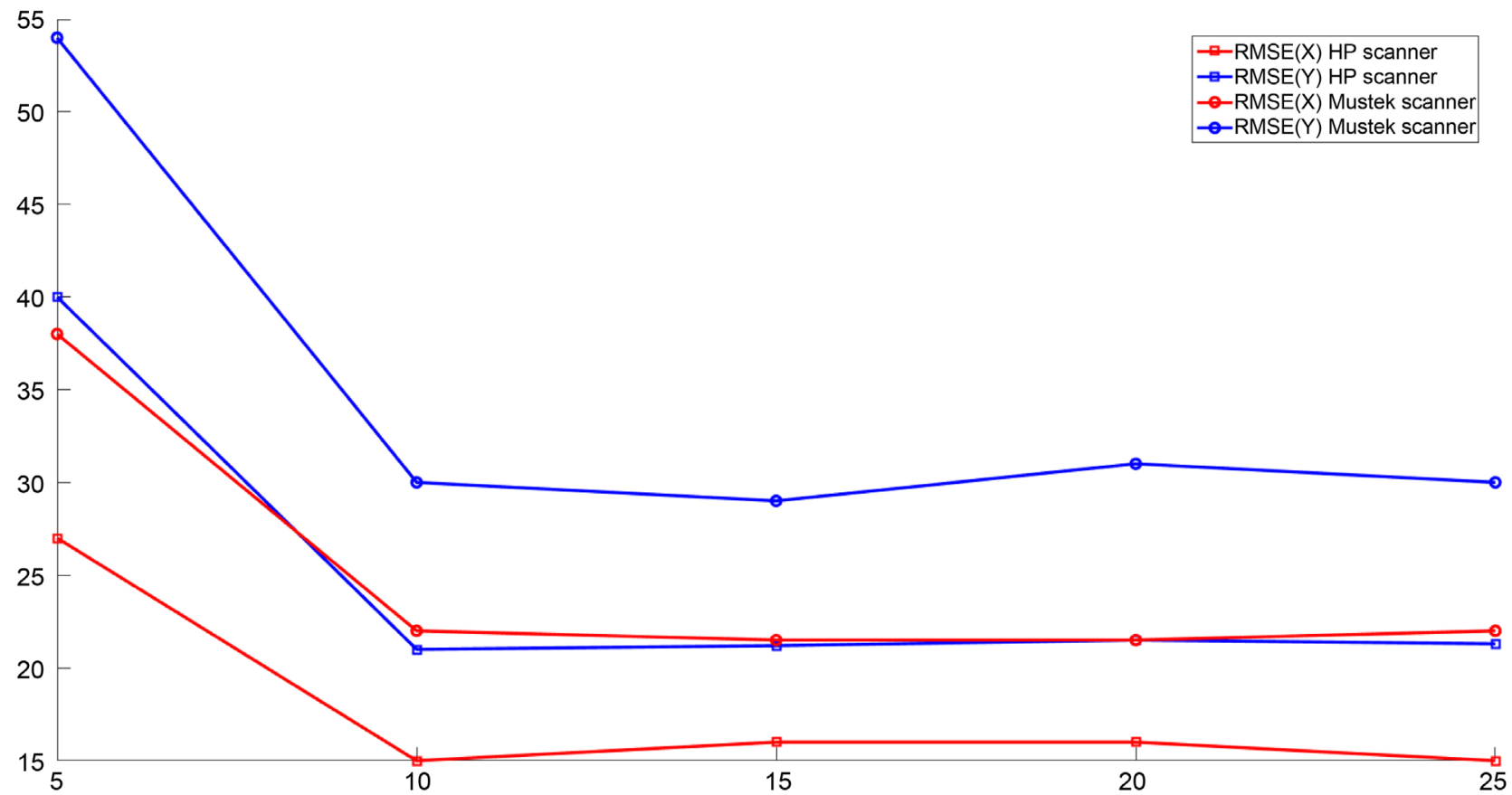

Figure 6. Changes in RMSEs (Vertical axis) as a function of the number of control points (horizontal axis).

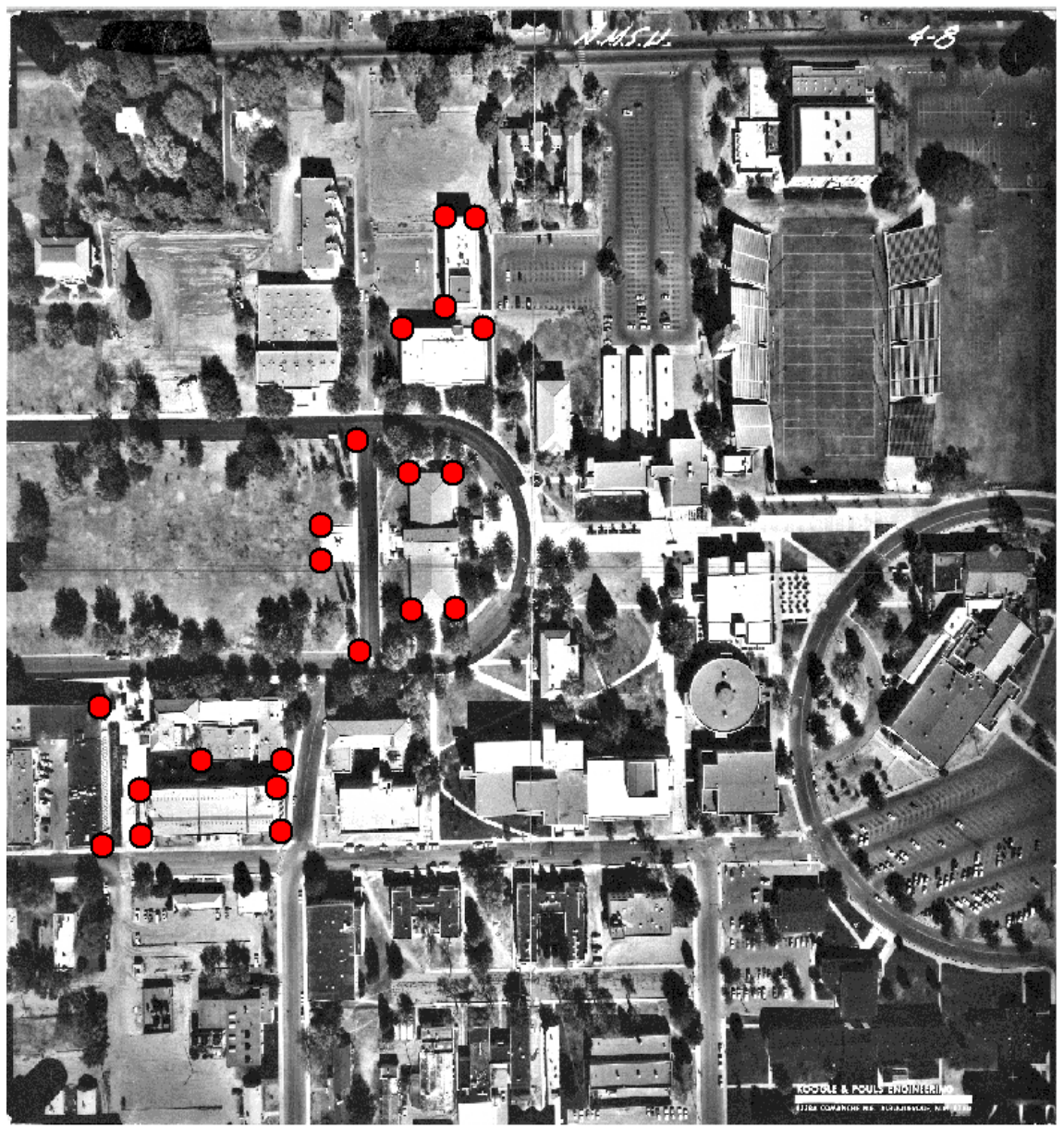

Figure 7. Ground points on a scanned image. 
Table 1. Average RMSEs $(\mu \mathrm{m})$ for the entire scanning glass with $2400 \mathrm{dpi}$.

\begin{tabular}{ccc}
\hline & HP scanner & Mustek scanner \\
\hline RMSE $X$ & 17.3 & 21.4 \\
RMSE $Y$ & 24.5 & 29.7 \\
\hline
\end{tabular}

Table 2. Distances between distances measured on the scanned photo and those measured on the ground.

\begin{tabular}{|c|c|c|c|c|}
\hline \multirow{2}{*}{ Resolution (dpi) } & \multirow{2}{*}{$\begin{array}{l}\text { Pixel size } \\
(\mu \mathrm{m})\end{array}$} & \multirow{2}{*}{$\begin{array}{l}\text { GSD } \\
(\mathrm{cm})\end{array}$} & \multicolumn{2}{|c|}{ Discrepancies $(\mathrm{cm})$} \\
\hline & & & HP scanner & Mustek scanner \\
\hline 600 & 40 & 10 & 32 & 47 \\
\hline 1200 & 20 & 5 & 22 & 29 \\
\hline 2400 & 10 & 2.5 & 21 & 31 \\
\hline
\end{tabular}

$1200 \mathrm{dpi}$ and the $2400 \mathrm{dpi}$ resolutions, discrepancies for both scanners are within the requirements for the ASPRS 1:1000 and 1:2000.

\section{Conclusion}

It is still imperative to scan historical maps and photos to build comprehensive geospatial databases. Scanners introduce geometrical distortions in the digitized coordinates. These distortions must be compensated before utilizing the digital data in further analysis. Several mathematical models are investigated to calibrate the flatbed scanners and to minimize the errors generated during the process. Many factors affect the RMSEs introduced by the scanner such as: the scanning resolution, the number and distribution of control points, and the mathematical model used in the transformation process. It was found that the mathematical model based on the projective transformation gives the best results for transforming the flatbed scanners coordinates to the actual hardcopy coordinates. At least ten control points are needed to perform reliable coordinate transformation with the projective transformation model. The distortion in the scanning direction is higher than that in the direction parallel to the linear CCD. As the resolution gets finer, less distortion is introduced. The ASPRS mapping standards could be readily achieved with a scanning resolution of $2400 \mathrm{dpi}$.

\section{References}

[1] Al-Ruzouq, R. (2012) Photogrammetry for Archaeological Documentation and Cultural Heritage Conservation. Special Applications of Photogrammetry, InTech. https://doi.org/10.5772/35314

[2] Elaksher, A.F. (2012) Potential of Using Automatically Extracted Straight Lines in Rectifying High-Resolution Satellite Images. International Journal of Remote Sensing, 33, 1-12. https://doi.org/10.1080/01431161.2010.489058

[3] Ahmad, A. (2011) Digital Mapping Using Low Altitude UAV. Pertanika Journal of Science and Technology, 19, 51-58.

[4] Mitishita, E., Habib, A., Centeno, J., Machado, A., Lay, J. and Wong, C. (2008) Photogrammetric and Lidar Data Integration Using the Centroid of a Rectangular 
Roof as a Control Point. The Photogrammetric Record, 23, 19-35. https://doi.org/10.1111/j.1477-9730.2008.00464.x

[5] Falkingham, P.L., Bates, K.T. and Farlow, J.O. (2014) Historical Photogrammetry: Bird's Paluxy River Dinosaur Chase Sequence Digitally Reconstructed as It Was Prior to Excavation 70 Years Ago. PloS ONE, 9, e93247.

https://doi.org/10.1371/journal.pone.0093247

[6] Nebiker, S., Lack, N. and Deuber, M. (2014) Building Change Detection from Historical Aerial Photographs Using Dense Image Matching and Object-Based Image Analysis. Remote Sensing, 6, 8310-8336. https://doi.org/10.3390/rs6098310

[7] Albuquerque, M.T.D., Sanz, G., Oliveira, S.F., Martínez-Alegría, R. and Antunes, I.M.H.R. (2013) Spatio-Temporal Groundwater Vulnerability Assessment-A Coupled Remote Sensing and GIS Approach for Historical Land Cover Reconstruction. Water Resources Management, 27, 4509-4526.

https://doi.org/10.1007/s11269-013-0422-0

[8] Nocerino, E., Menna, F. and Remondino, F. (2012) Multi-Temporal Analysis of Landscapes and Urban Areas. International Archives of Photogrammetry, Remote Sensing \& GIS, 85-90.

[9] De Rose, R.C. and Basher, L.R. (2011) Measurement of River Bank and Cliff Erosion from Sequential LIDAR and Historical Aerial Photography. Geomorphology, 126, 132-147. https://doi.org/10.1016/j.geomorph.2010.10.037

[10] Mackey, B.H. and Roering, J.J. (2011) Sediment Yield, Spatial Characteristics, and the Long-Term Evolution of Active Earthflows Determined from Airborne LiDAR and Historical Aerial Photographs, Eel River, California. Geological Society of America Bulletin, 123, 1560-1576. https://doi.org/10.1130/B30306.1

[11] Sevara, C. (2016) Capturing the Past for the Future: An Evaluation of the Effect of Geometric Scan Deformities on the Performance of Aerial Archival Media in Image-Based Modelling Environments. Archaeological Prospection, 23, 325-334. https://doi.org/10.1002/arp.1539

[12] Gruber, M. and Leberl, F. (2001) Description and Evaluation of the High Quality Photogrammetric Scanner UltraScan 5000. ISPRS Journal of Photogrammetry and Remote Sensing, 55, 313-329. https://doi.org/10.1016/S0924-2716(01)00024-7

[13] Mitrovic, M., Cvijetinovic, Z. and Mihajlovic, D. (2004) Procedures and Experiences on Using Desktop Scanner for Orthophoto Production. 20th ISPRS Congress, Istanbul, 12-23 July 2004, 53-58.

[14] Göröcs, Z. and Ozcan, A. (2014) Biomedical Imaging and Sensing using Flatbed Scanners. Lab on a Chip, 14, 3248-3257. https://doi.org/10.1039/C4LC00530A

[15] Gergel, S.E., Coops, N.C. and Morgan, J.L. (2010) Aerial Photography: A Rapidly Evolving Tool for Ecological Management. Bioscience, 60, 47-59.

[16] Yousefhussien, M., Easton Jr., R.L., Ptucha, R.W., Shaw, M.Q., Bradburn, B.M., Wagner, J., Saber, E., et al. (2015) Flatbed Scanner Simulation to Analyze the Effect of Detector's Size on Color Artifacts. Proceedings of the SPIE, 9401, 94010H.

[17] Zheleznyak, A.G. and Sidorov, V.G. (2015) Flatbed Scanner as an Instrument for Physical Studies. St. Petersburg Polytechnical University Journal: Physics and Mathematics, 1, 134-141. https://doi.org/10.1016/j.spjpm.2015.04.001

[18] Williams, G.J., Maddox, B.R., Chen, H., Kojima, S. and Millecchia, M. (2014) Calibration and Equivalency Analysis of Image Plate Scanners A. Review of Scientific Instruments, 85, 11E604. https://doi.org/10.1063/1.4886390

[19] Baltsavias, E.P., Haering, S. and Kersten, T.P. (1997) Geometric and Radiometric 
Performance Evaluation of the Leica/Helava DSW200 Photogrammetric Film Scanner. Proceedings of the SPIE, 3174, 157-173.

[20] Bethel, J. (1994) Calibration of a Photogrammetric Image Scanner. Technical Papers, ASPRS/ ACSM Annual Convention, Reno, 25-28 April 1994, Vol. 1, 81-88.

[21] Ruzgienė, B., Bagdžiūnaitè, R. and Ruginyte், V. (2012) Scanning Aerial Photos using a Non-Professional Scanner. Geodesy and Cartography, 38, 118-121. https://doi.org/10.3846/20296991.2012.728901

[22] El-Ashmawy, K.L. (2014) Using Low Cost DeskTop Publishing (DTP) Scanners for Aerial Photogrammetry. Journal of Geosciences and Geomatics, 2, 107-113.

[23] De Vicente, J., Sánchez-Pérez, A.M., Maresca, P., Caja, J. and Gómez, E. (2015) A Model to Transform a Commercial Flatbed Scanner into a Two-Coordinates Measuring Machine. Measurement, 73, 304-312. https://doi.org/10.1016/j.measurement.2015.05.029

[24] Jones, M.P., Callahan, R.N. and Bruce, R.D. (2012) Dimensional Measurement Variation of Scanned Objects using Flatbed Scanners. Journal of Technology, Management \& Applied Engineering, 28, 1-12.

[25] Wyatt, M. and Nave, G. (2017) Evaluation of Resolution and Periodic Errors of a Flatbed Scanner Used for Digitizing Spectroscopic Photographic Plates. Applied Optics, 56, 3744-3749. https://doi.org/10.1364/AO.56.003744

[26] De Vicente, J., Sánchez-Perez, A.M., Berzal, M., Maresca, P. and Gómez, E. (2013) Uncertainty in Ellipse Fitting using a Flatbed Scanner: Development and Experimental Verification. Measurement Science and Technology, 25, Article ID: 015005. https://doi.org/10.1088/0957-0233/25/1/015005

[27] Kangasrääsiö, J. and Hemming, B. (2009) Calibration of a Flatbed Scanner for Traceable Paper Area Measurement. Measurement Science and Technology, 20, Article ID: 107003. https://doi.org/10.1088/0957-0233/20/10/107003

[28] Poppinga, D., Schoenfeld, A.A., Doerner, K.J., Blanck, O., Harder, D. and Poppe, B. (2014) A New Correction Method Serving to Eliminate the Parabola Effect of Flatbed Scanners Used in Radiochromic Film Dosimetry. Medical Physics, 41, Article ID: 021707. https://doi.org/10.1118/1.4861098

[29] Meng, G., Wang, Y., Qu, S., Xiang, S. and Pan, C. (2014) Active Flattening of Curved Document Images via Two Structured Beams. Proceedings of the IEEE Conference on Computer Vision and Pattern Recognition, Columbus, 23-28 June 2014, 3890-3897. https://doi.org/10.1109/CVPR.2014.497

[30] Suzuki, Y., Yamashita, A. and Kaneko, T. (2007) Correction of Geometric and Photometric Distortion of Document Images using a Stereo Camera System. Proceedings of the IAPR Conference on Machine Vision Applications, Tokyo, 16-18 May 2007, 215-218.

[31] Zhang, L., Zhang, Z., Tan, C.L. and Xia, T. (2005) 3D Geometric and Optical Modeling of Warped Document Images from Scanners. Computer Society Conference on Computer Vision and Pattern Recognition, San Diego, 20-25 June 2005, Vol. 1, 337-342.

[32] He, Y., Pan, P., Xie, S., Sun, J. and Naoi, S. (2013) A Book Dewarping System by Boundary-Based 3D Surface Reconstruction. 12th International Conference on Document Analysis and Recognition, Washington DC, 25-28 August 2013, 403-407.

[33] Leica (2017).

https://leica-geosystems.com/services-and-support/product-services/film-scanner-s upport 
[34] Baltsavias, E.P. and Patias, P. (1995) On the Use of DTP Scanners for Cartographic Applications.

[35] ASPRS (1990) ASPRS Accuracy Standards for Large-Scale Maps. http://www.asprs.org/a/society/committees/standards/1990_jul_1068-1070.pdf 